\title{
Rates of Criminal Legal System Involvement Among Patients Who Are Discharged Against Medical Advice
}

\author{
Nora Abo-Sido, $M D^{7}$, Lisa Simon, $D M^{1,2}$, and Matthew Tobey, MD MPH ${ }^{1,3}$ \\ ${ }^{1}$ Harvard Medical School, Boston, MA, USA; ${ }^{2}$ Department of Oral Health Policy and Epidemiology, Harvard School of Dental Medicine, Boston, MA, \\ USA; ${ }^{3}$ Massachusetts General Hospital, Boston, MA, USA.
}

KEY WORDS: incarceration; against medical advice; access to care; risk factors; disparities.

J Gen Intern Med 33(10): 1616-7

DOI: $10.1007 / \mathrm{s} 11606-018-4541-4$

(C) Society of General Internal Medicine 2018

\section{INTRODUCTION}

Patients discharged from the hospital against medical advice (DAMA) are at elevated risk of poor health outcomes, including re-hospitalization and death. ${ }^{1}$ DAMA patients are more likely to be young, male, have non-white ethnicity, have low income, experience homelessness, and to suffer from mental illness and substance use disorders. ${ }^{2}, 3$

The US criminal legal system incarcerates more than 2 million Americans, disproportionately young men of color. People with a history of incarceration have poorer health, are more likely to be hospitalized after release, and are more likely to utilize the emergency department for care. ${ }^{5}$ Many patients with a history of incarceration report experiencing discrimination by healthcare workers, which may compound feelings of disempowerment and dissatisfaction with care that lead to DAMA. ${ }^{6}$ The purpose of this study was to evaluate whether
DAMA was associated with a history of criminal legal involvement (CLI) among patients at a large urban tertiarycare medical center in the northeastern USA.

\section{METHODS}

We performed a chart review at a tertiary-care hospital of all patients with DAMA from $9 / 1 / 2015$ to $8 / 31 / 2016$. The study was approved by the Partners HealthCare Institutional Review Board (\#2016P002191).

DAMA charts were reviewed for variables including demographics, medical history, behavioral health history, social history, and history of CLI. For appropriate variables, a freetext search of the chart was performed using QPID Health (QPID Health Inc). The tool does not search scanned outside records.. We analyzed the results using STATA (Version 15; Statacorp).

\section{RESULTS}

DAMA occurred in 297 patients over a 1-year period, of whom $147(49 \%)$ had a history of CLI documented in their

Table 1 Characteristics of Patients Discharged Against Medical Advice, Overall, and by Presence of Criminal Legal Involvement

\begin{tabular}{|c|c|c|c|c|c|c|c|}
\hline & \multicolumn{2}{|c|}{ Overall $N=297$} & \multicolumn{2}{|c|}{ CLI $N=147$} & \multicolumn{2}{|c|}{ No CLI $N=150$} & \multirow{2}{*}{$\frac{\text { CLI v No CLI }}{p \text { value }}$} \\
\hline & Mean $/ \%$ & St. dev. & Mean/\% & St. dev. & Mean/\% & St. dev. & \\
\hline Age & 49.3 & 16.0 & 46.5 & 13.0 & 52.1 & 18.1 & 0.002 \\
\hline Female & $30 \%$ & & $18 \%$ & & $41 \%$ & & $<0.001 *$ \\
\hline White & $79 \%$ & & $82 \%$ & & $77 \%$ & & 0.36 \\
\hline$>1$ AMA from inpt/ED & $44 \%$ & & $59 \%$ & & $29 \%$ & & $<0.001 *$ \\
\hline LOS $48 \mathrm{~h}$ or less & $46 \%$ & & $48 \%$ & & $45 \%$ & & 0.61 \\
\hline Medicine service & $82 \%$ & & $90 \%$ & & $73 \%$ & & $<0.001 *$ \\
\hline \# medical diagnoses & 5.0 & 3.6 & 4.2 & 2.7 & 5.8 & 4.1 & $<0.001 *$ \\
\hline Diagnosis of chronic pain & $27 \%$ & & $22 \%$ & & $32 \%$ & & 0.047 \\
\hline History or current IVDU & $22 \%$ & & $36 \%$ & & $8 \%$ & & $<0.001 *$ \\
\hline Opioid use disorder & $34 \%$ & & $51 \%$ & & $17 \%$ & & $<0.001 *$ \\
\hline Alcohol use disorder & $33 \%$ & & $46 \%$ & & $20 \%$ & & $<0.001 *$ \\
\hline Smoking & $63 \%$ & & $82 \%$ & & $44 \%$ & & $<0.001 *$ \\
\hline Withdrawal & $29 \%$ & & $43 \%$ & & $16 \%$ & & $<0.001 *$ \\
\hline Other behavioral health diagnosis & $61 \%$ & & $72 \%$ & & $51 \%$ & & $<0.001 *$ \\
\hline Currently homeless & $35 \%$ & & $55 \%$ & & $16 \%$ & & $<0.001 *$ \\
\hline
\end{tabular}

CLI criminal legal involvement, ED emergency department, LOS length of stay of inpatient admission, IVDU intravenous drug use Statistical testing, Chi-square test for all measures reported as percentages; Two-tailed unpaired Student's T test with unequal variance for data reported as mean/st. dev.

*Significant

Published online June 29, 2018 
Table 2 Specific Documentation of Criminal Legal Involvement Among Patients Discharged Against Medical Advice*

\begin{tabular}{lll}
\hline \hline & $\boldsymbol{N}$ & $\boldsymbol{\%}$ \\
\hline Any criminal legal involvement & 147 & - \\
Any Incarceration & 117 & 80 \\
Jail & 66 & 45 \\
Prison & 35 & 24 \\
In custody during at least one hospitalization & 9 & 6 \\
Probation & 19 & 12 \\
Parole & 6 & 4 \\
Open case, not in custody/incarcerated & 18 & 12 \\
Alcohol-related arrest only & 4 & 3 \\
\hline
\end{tabular}

*Some patients met multiple categories; e.g., a patient with a history of a prison stay who is currently on parole is listed in both

chart. Table 1 compares those individuals with DAMA with and without CLI. DAMA patients with a history of CLI were younger and more likely to be male, have a history of homelessness, have multiple DAMAs, receive care on the medicine service, have an injection drug use history, have an opioid use disorder history, have an alcohol use disorder history, smoke, be experiencing withdrawal, have fewer medical diagnoses, and have more behavioral health diagnoses $(p<0.01$ for each comparison).

Documentation allowed for partial or complete clarification of the nature of CLI in 142 of 147 individuals (Table 2). Of these, $117(80 \%)$ had a history of incarceration or were currently incarcerated, 66 (45\%) had a history of jail stays, and 35 $(24 \%)$ had a history of prison stays. Six (4\%) were on parole and $19(12 \%)$ were on probation.

\section{DISCUSSION}

Our analysis found that half of patients at our institution with DAMA have experienced CLI. Patients with CLI who left DAMA shared traits with individuals with CLI in the community, including younger age, higher rates of substance use disorders, and a higher burden of behavioral health disorders. They were also more likely to have multiple episodes of DAMA. Study limitations include that we were unable to confirm rates of CLI, which may be underreported in the chart due to stigma and lack of provider awareness. The rate of CLI was very high in our inner-city population, which may not be true in other cohorts. The CLI population in this study was more likely to be white than the national incarcerated population, which may be related to our institution's inpatient demographics, thereby limiting generalizability.

Individuals who have previously experienced CLI or incarceration may feel more acutely disempowered during a hospital admission and may have outstanding legal concerns. Knowledge of the association between CLI and DAMA may help providers reduce DAMA rates through improved communication and anticipation of social or behavioral needs.

Corresponding Author: Lisa Simon, DMD; Department of Oral Health Policy and EpidemiologyHarvard School of Dental Medicine, Boston, MA, USA (e-mail: Lisa_simon@hsdm.harvard.edu).

\section{Compliance with ethical standards:}

Conflicts of interest: The authors declare that they do not have a conflict of interest.

\section{REFERENCES}

1. Glasgow JM, Vaughn-Sarrazin M, Kaboli PJ. Leaving against medical advice (AMA): risk of 30-day mortality and hospital readmission. J Gen Intern Med. 2010;25(9):926-929. doi:https://doi.org/10.1007/s11606010-1371-4.

2. Fiscella $\mathbf{K}$, Meldrum S, Barnett $\mathbf{S}$. Hospital discharge against advice after myocardial infarction: deaths and readmissions. Am J Med. 2007;120(12):1047-1053. doi:https://doi.org/10.1016/j.amjmed.2007. 08.024 .

3. Spooner KK, Salemi JL, Salihu HM, Zoorob RJ. Discharge against medical advice in the United States, 2002-2011. Mayo Clin Proc. 2017;92(4):525-535. doi:https://doi.org/10.1016/j.mayocp.2016.12.022.

4. Kaeble D, Glaze L. Correctional Populations in the United States, 2015, US Dep Justice, Off Justice Programs, Bur Justice Stat. 2016.

5. Winkelman TNA, Choi H, Davis MM. The Affordable Care Act, Insurance Coverage, and Health Care Utilization of Previously Incarcerated Young Men: 2008-2015. Am J Public Health. 2017;107(5):807-811. doi:https:// doi.org/10.2105/AJPH.2017.303703.

6. Frank JW, Wang EA, Nunez-Smith M, Lee H, Comfort M. Discrimination based on criminal record and healthcare utilization among men recently released from prison: a descriptive study. Heal justice. 2014;2:6. doi:https://doi.org/10.1186/2194-7899-2-6. 